\title{
Potential activity of GIT-27 against renal ischemia reperfusion injury: an experimental study in male rats \\ Najah Hadi $^{1 *}$, Huda jabber
}

\begin{abstract}
The detail mechanisms in which renal ischemia reperfusion IRI happens are still indistinct. Various elements required in the pathogenesis include oxidative stress, inflammation, cellular necrosis, apoptosis, renal pathophysiological changes etc. To understand the pathway of ischemia reperfusion in renal we tested the hypothesis that GIT-27 attenuated renal ischemia reperfusion injury as specific Toll-like receptor inhibitors. Adult (3 to 5 month) male Sprague Dawely rats, and their weights ranged from 180 to $390 \mathrm{gm}$, were pre-treated with Git-27 intra peritoneal, plasma NGAL and cytokines mediator's in plasma and renal were analyzed by enzyme-linked immunosorbent assay (ELISA). And the pathological changes and cells injury in the renal were examined using hematoxylin and Eosin staining. Improvement of renal ischemia reperfusion by GIT-27 resulted in improved renal function and greater reductions in inflammatory mediators, and kidney injury. Taken together, GIT-27 significantly improved renal function following I/R through down-regulation of Tolllike receptor and serve as a potential therapeutic in ischemia reperfusion induced acute kidney injury.
\end{abstract}

Keywords: Renal ischemia reperfusion; Git-27; NGAL

"Corresponding Author: Najah Hadi: dr.najahhadi@yahoo.com

Author Affiliations

${ }^{1}$ Department of pharmacology, College of Medicine, University of Kufa

Received June 22, 2016; accepted September 21, 2016; published October 29, 2016

Copyright $\odot 2016 \mathrm{NH}$, et al. This is article distributed under the terms of the Creative Commons Attribution License (http://creativecommons.org), which permits unrestricted use, distribution, and reproduction in any medium, provided the original work is properly cited.

\section{Introduction}

Renal ischemia reperfusion (I/R) is an inflammatory process that causes acute kidney injury (AKI). AKI may not just happen with regards to kidney transplantation in which $\mathrm{I} / \mathrm{R}$ is inescapable, but on the other hand is an outcome of hindered kidney perfusion e.g. amid real surgery or sepsis. For the kidney, IR is either because of heart failure (systemic hypo perfusion), surgical intercessions prompting nearby renal hypo perfusion, for example, and aortic cross-clipping, incomplete nephrectomy and in addition transplantation. AKI is freely connected 
with expanded unpleasantness and mortality and additionally expanded length of clinic stays [1]. AKI is freely connected with a 2-to 5-fold expanded danger of death [2]. The detail mechanisms in which renal IRI happens are still indistinct. Various elements required in the pathogenesis include oxidative stress [3] inflammation [4] cellular necrosis [5], apoptosis [6] etc. Taking into account these conceivable components, a great deal of competitor medicines have been accounted for protective affecting renal IRI [7, 8, 9, 10]. GIT-27unusual immune modulator, is under development for the management of rheumatoid arthritis [11]. Suppresses TNF- $\alpha$ emission by means of intervention of macrophage toll-like receptor (TLR) 4 and TLR 2/6 signaling pathway. Toll-like receptors (TLRs) are pattern-recognition receptors that principally work as initiators of the distinguishing innate immune response [12].Additionally decreases the release of proinflammatory cytokines IL1- $\beta$, and IFN- $\gamma$. Anti diabetogenic; anticipates IL- $\beta$ and IFN- $\gamma$-affected pancreatic islet cell demise in vitro. The flexibility of the agent's action, its high efficacy, and low toxicity and suggests that GIT-27 is a candidate for anticancer drug of the future [13].

\section{Materials and method}

\section{Experimental animals}

Adult (3 - 5 months) male Sprague Dawely rats, and their weights ranged from 180 to 390gm gained from Animal Resource Center, establishment of collection life examination and treatment of unprofitability, Al-Nahrain
University. Rats were adapted for two weeks in light/dark cycle (12:12-h) with availability of free access to water and regular chow diet in animal house of Medical College, Kufa University and this investigation conforms to according to the Guide for the Care and Use of Laboratory Animals.

\section{Renal ischemia reperfusion procedure} in rats

Renal ischemia reperfusion ( $\mathrm{R} \mathrm{I/R}$ ) was established to induce acute kidney injury in murine as described previously [14]. In brief, rat was anesthetized by i.p. injection mixture of ketamine and xylazine (Dreieich, Leverkusen, Germany). A 2-cm abdominal midline incision was performed to exposed kidneys, did already right kidney nephrectomy. Then we clamped left kidney for 30 minute and reperfused for 3 hour using a 4.0 surgical suture (Norderstedt, Germany). Rats monitoring during reperfusion time for various signs of sickness. Sham rat received anesthesia and laparotomy without renal I/R served as the surgical control group. The animals were assigned to the following experimental groups: sham group, vehicle group, $\mathrm{R} \quad \mathrm{I} / \mathrm{R}$ group, $\mathrm{RI} / \mathrm{R}+$ Pretreated with GIT-27 $(n=7$ in each group).

\section{Collection of samples}

The samples of blood were drawn directly from the heart using needle, with heparin as anticoagulant and stored at $4^{\circ} \mathrm{C}$. The blood centrifuged at $3000 \mathrm{rpm}$ for $15 \mathrm{~min}$ at $4^{\circ} \mathrm{C}$, and collected plasma stored at $-20^{\circ} \mathrm{C}$. Also at $3000 \mathrm{rpm}$ for 10 minutes and collected serum stored at $-20^{\circ} \mathrm{C}$ until 
used for further analyses for the right kidney of the rat was excised. Renal tissues were cut into two parts: upper half of kidney was snap-frozen (-70) until use, while the remaining parts were used for histological analysis.

\section{ELISA}

The upper part of kidney treated in PBS containing $0.5 \%$ Triton X100 with a protease inhibitor cocktail, tissue was homogenized and the supernatant used to quantify (interlukin-18 level) in addition to the plasma neutrophil gelatinase associated lipocalin (NGAL) and serum(urea, creatinine, TNF alpha level) according the instruction of commercial ELISA kits (R\&D Systems). The spectrophotometry of micro plate reader (Bio-Rad Laboratories, USA) was used to determine the absorbance of standards and samples at $450 \mathrm{~nm}$.

\section{Histological examination}

The renal tissue samples were fixed in $4 \%$ paraformaldehyde for $24 \mathrm{~h}$, as described previously [15]. Briefly, 5 $\mu \mathrm{m}$ sections in thickness of renal tissue were paraffin embedded stained with the hematoxylin and eosin (H\&E) through the standard procedure. The scoring of renal injury was examined according to the protocol of McWhinnie [16], quantitative measurements of tissue damage by a blinded observer. Tubular damage was defined as tubular epithelial swelling, loss of brush border, vacuolar degeneration, necrotic tubules, cast formation, and desquamation. The degree of kidney damage was estimated at $\times 200$ magnification, using five randomly selected fields for each animal, by the following criteria were used to scoring the renal injury: 0 , normal; 1 , area of damage $<25 \%$ of tubules; 2, damage involving 25-50\% of tubules; 3, damage involving 50$75 \%$ of tubules; and $4,75-100 \%$ of the area being affected.

\section{Statistical analysis}

The data statistically analyzed by using Kolmogorov-Smirnova test and Shapiro-Wilk test to investigate differences between rats. Statistically the present data significance was defined as $\mathrm{P}<0.05$. The results were presented in the form of a table and a graph for each variable among different groups. P1 for control versus sham, P2 for control versus vehicle, P3 for control versus GIT-27.

\section{Results}

GIT-27 improved renal function after $I / R$ injury

To investigate the treatment effects of GIT-27on renal function following acute kidney injury by using renal $I / R$ protocol. Renal function dropped in $\mathrm{I} / \mathrm{R}$ and vehicle $(P<0.05)$ (Tab. 1A, B) (Fig. 1A, B) Furthermore, GIT-27 pretreated groups improved renal function through increased renal output $(\mathrm{P}<$ $0.05)$. 
Table 1A: Mean serum urea in all groups

\begin{tabular}{|r|c|c|c|c|c|}
\hline Group & Mean & SD & P1 & P2 & P3 \\
\hline Sham & 45.40 & 6.38 & & & \\
\cline { 1 - 3 } Control & 75.51 & 15.84 & & & \\
\cline { 1 - 3 } Vehicle & 70.10 & 5.28 & \multirow{2}{*}{0.003} & 0.277 & 0.009 \\
\cline { 1 - 3 } GIT-22 & 49.38 & 5.32 & & & \\
\hline
\end{tabular}

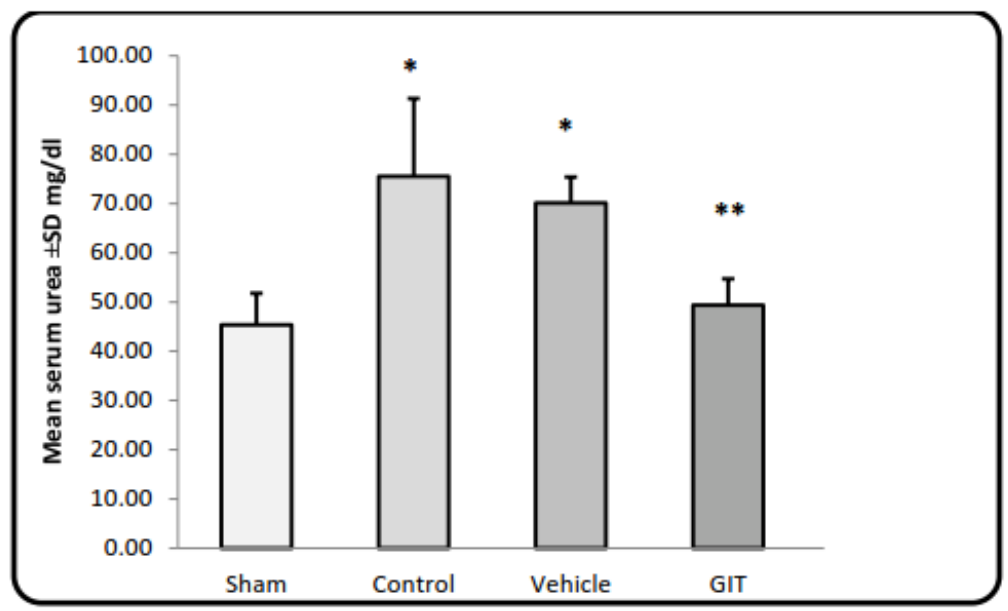

\section{Figure 1A.}

The mean serum urea $(\mathrm{mg} / \mathrm{dl})$ among four $(n=7)$ experimental groups. GIT-27(25 mg/kg) suppressed the level of serum urea after renal I/R injury. Data are expressed as mean \pm standard error; ${ }^{*} P<0.05$ versus corresponding sham; ${ }^{* *} P<0.05$ versus I/R rats.

Effective role of GIT-27 on proInflammatory cytokines after renal $I / R$ We next investigated the importance effects of GIT-27 on the local and systemic pro-inflammatory responses during renal $I / R$. At the end of the experiment ( 3 hour after renal I/R), the levels of pro-inflammatory mediators including (TNF- $\alpha$, IL-18) in serum and renal tissue are measured by ELISA according to manufacture protocol. The resulted data showed that all proinflammatory cytokines are increased in both renal I/R and vehicle treatment rat group compared with sham group $(\mathrm{P}<$ 0.05 ) in both serum and renal tissue (Tab. 2A, B) (Fig. 2A, B).

\section{Table 2A.}

Mean serum TNF- $\alpha$ in all groups

\begin{tabular}{|l|c|c|c|c|c|}
\hline Variable & Mean & SD & P1 & P2 & P3 \\
\cline { 1 - 3 } Sham & 25.64 & 4.63 & & & \\
\cline { 1 - 3 } Control & 45.33 & 35.39 & & & \\
\cline { 1 - 3 } Vehicle & 40.41 & 3.45 & 0.013 & 0.047 & 0.565 \\
\cline { 1 - 3 } GIT-27 & 31.24 & 3.66 & & & \\
\hline
\end{tabular}




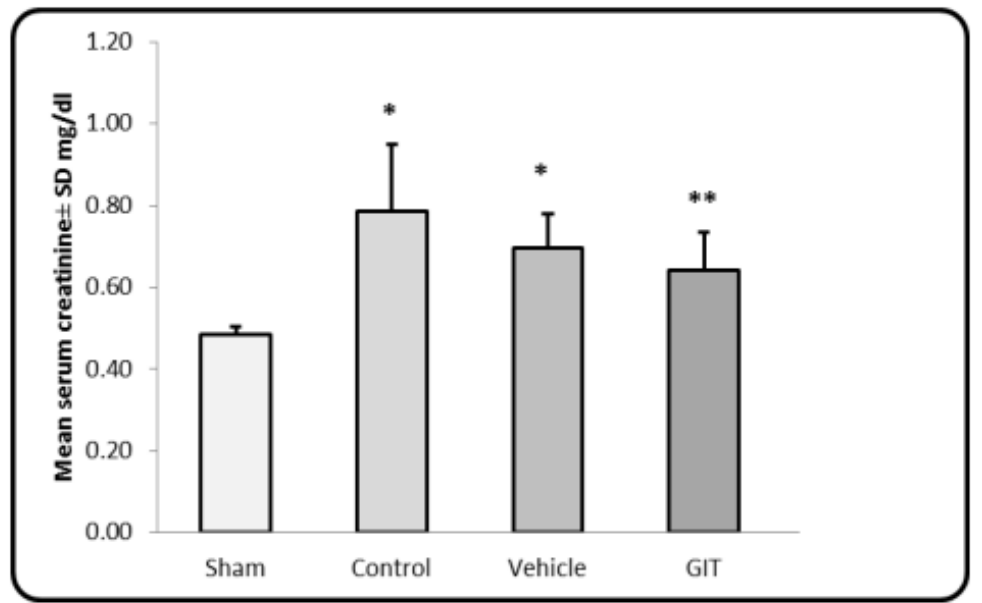

\section{Figure 2A.}

The mean serum TNF- $\alpha(\mathrm{pg} / \mathrm{ml})$ among four $(\boldsymbol{n}=7)$ experimental groups. GIT-27 $(25 \mathrm{mg} / \mathrm{kg})$ suppressed the level of serum TNF- $\alpha$ after renal I/R injury. Data are expressed as mean \pm standard error; ${ }^{*} P<0.05$ versus corresponding sham; ${ }^{* *} P<0.05$ versus I/R rats.

Table 2B.

Mean tissue IL-18 in all groups

\begin{tabular}{|l|l|l|l|l|l|}
\hline Variable & Mean & SD & P1 & P2 & P3 \\
\hline Sham & 62.71 & 4.35 & & & \\
\cline { 1 - 3 } Control & 378.29 & 5.25 & & & \\
\cline { 1 - 3 } Vehicle & 364.43 & 10.71 & 0.002 & 0.018 & 0.002 \\
\cline { 1 - 3 } GIT-27 & 152.71 & 4.42 & & & \\
\cline { 1 - 3 }
\end{tabular}

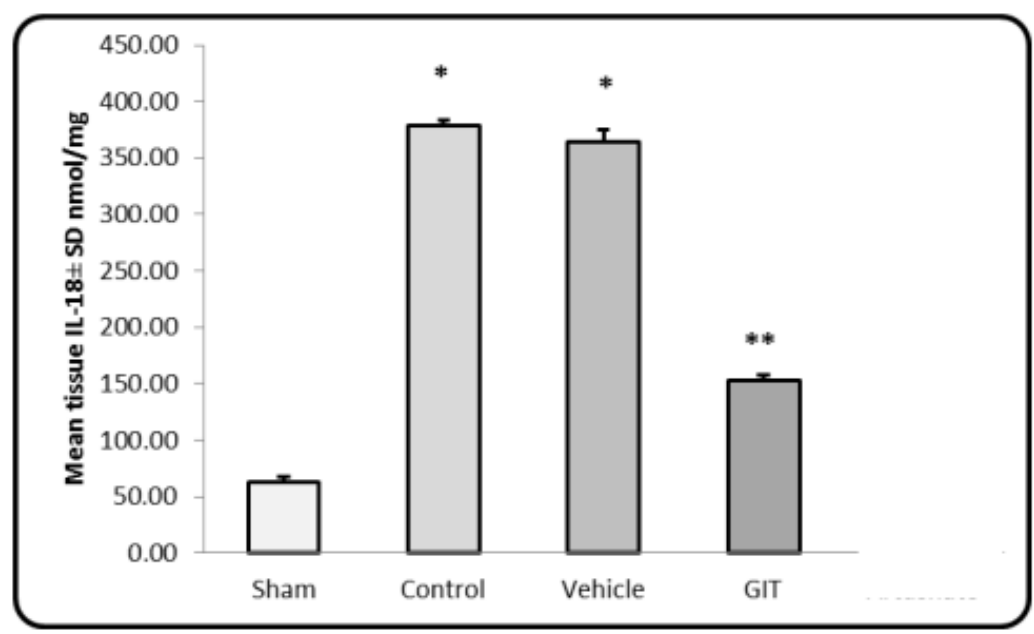

Figure 2B.

The mean tissue IL-18 (n mol/mg) among four ( $n=7)$ experimental groups. GIT-27(25 mg/kg) suppressed the level of tissueIL-18after renal I/R injury. Data are expressed as mean \pm standard error; ${ }^{*} P<0.05$ versus corresponding sham; ${ }^{* *} P<0.05$ versus $\mathrm{I} / \mathrm{R}$ rats. 
GIT-27 suppresses the expression level of NGAL

Renal I/R lead to upstream release of NGAL expression in plasma. We assayed the expression of NGAL in plasma by ELISA. (Tab. 3) (Fig. 3) showed that the levels of NGAL are markedly increased by renal $\mathrm{I} / \mathrm{R}$, whereas GIT-27 treatment attenuates plasma NGAL levels $(P<0.05)$.

\section{Table 3.}

Mean plasma Tissue NGAL in all groups

\begin{tabular}{|l|l|l|l|l|l|}
\hline Variable & Mean & SD & P1 & P2 & P3 \\
\cline { 1 - 3 } Sham & 24.54 & 1.91 & & & \\
\cline { 1 - 3 } Control & 188.00 & 2.58 & & & \\
\cline { 1 - 3 } Vehicle & 184.43 & 8.56 & 002 & 0.847 & 0.002 \\
\cline { 1 - 3 } GIT-27 & 76.00 & 3.51 & & & \\
\hline
\end{tabular}

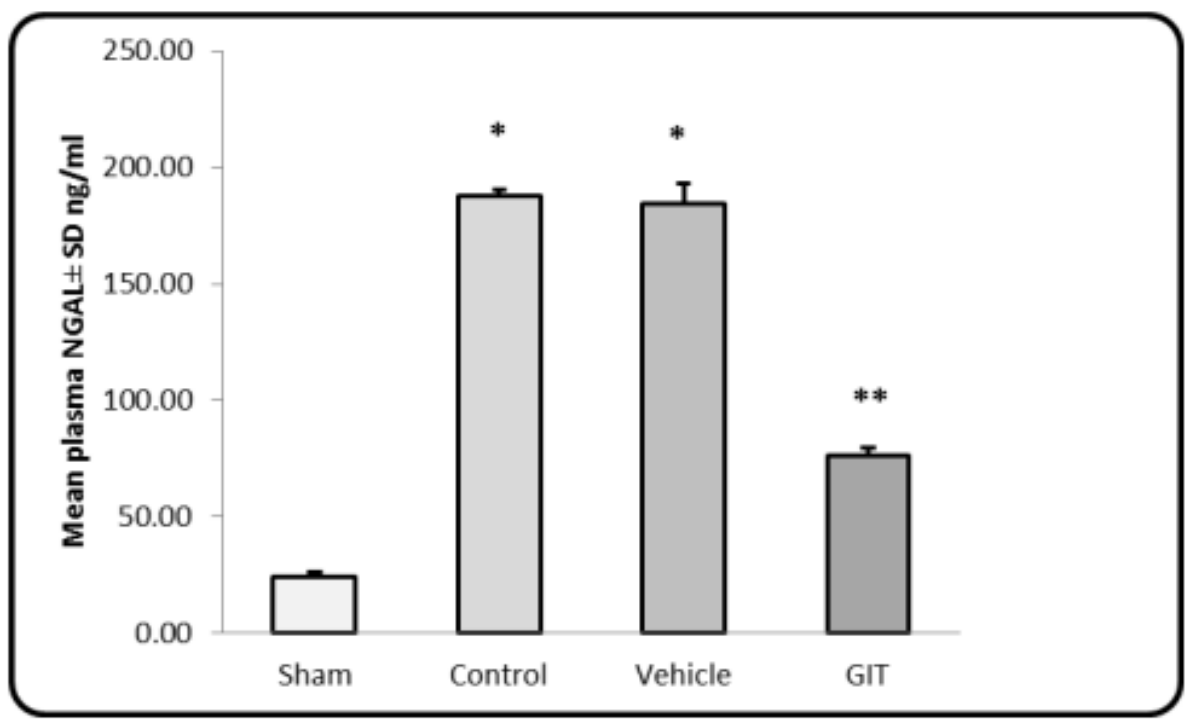

Figure 3.

The mean plasma NGAL $(\mathrm{ng} / \mathrm{ml})$ among four $(n=7)$ experimental groups.

GIT-27(25 mg/kg) suppressed the level of tissue NGAL after renal I/R injury. Data are expressed as mean \pm standard error; ${ }^{*} P<0.05$ versus corresponding sham; ${ }^{* *} P<0.05$ versus I/R rats

Git-27 treatment improved histopathological changes after renal $I / R$

Histologically, renal tissue from $\mathrm{I} / \mathrm{R}$ or vehicle rat after 3 hour of reperfusion period (Fig. 5A, B, C) revealed renal injury by quantitative measurements of tissue damage by a blinded observer.
Tubular damage was defined as tubular epithelial swelling, loss of brush border, vacuolar degeneration, necrotic tubules, cast formation, and desquamation. The degree of kidney damage was estimated at $\times 200$ magnification,. While the histopathological changes in GIT-27 
treated group showed mild cellular alterations (Fig. 6A, B). According to McWhinnie scoring system; 0, normal; 1 , area of damage $<25 \%$ of tubules; 2 , damage involving $25-50 \%$ of tubules; 3 , damage involving $50-75 \%$ of tubules; and 4, 75-100\% of the area being affected. Mean histological score of GIT-27 group were significantly lower than that of control group $(P<0.05)$ (Fig. 4).

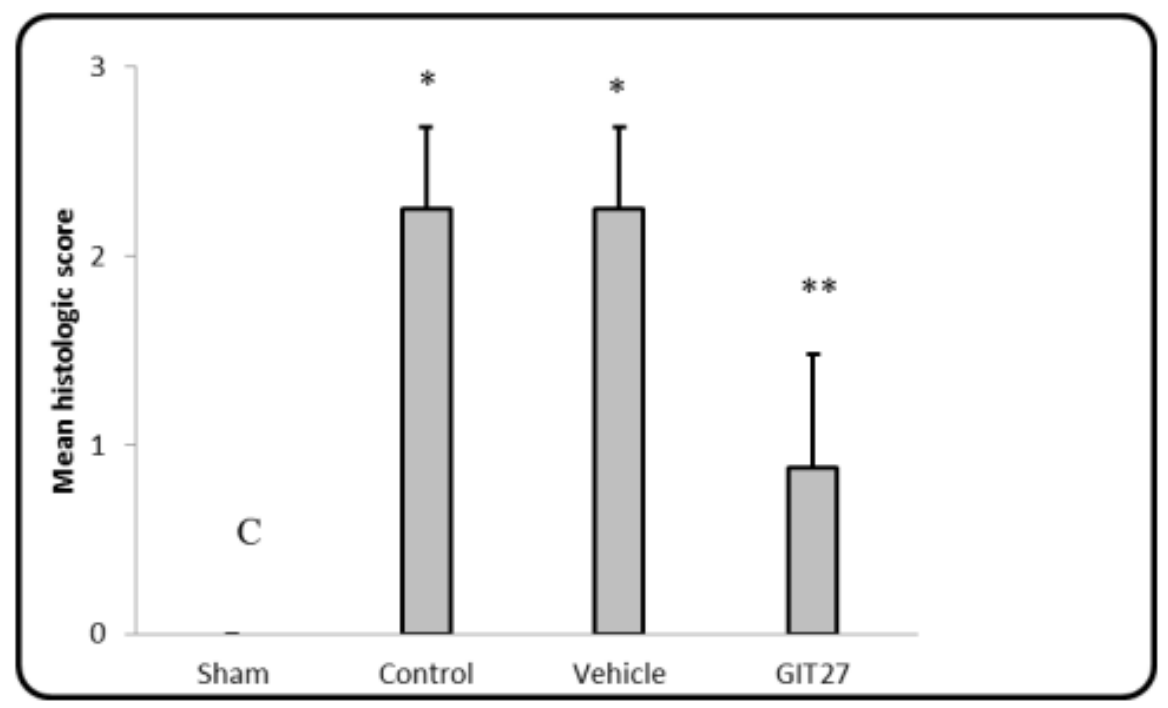

Figure 4.

The mean histological score among four ( $n=7)$ experimental groups. GIT-27 (25 mg/kg) treatment improved histopatho-logical changes after renal I/R. Data are expressed as mean \pm standard error; ${ }^{*} P$ $<0.05$ versus corresponding sham; ${ }^{* *} P<0.05$ versus $\mathrm{I} / \mathrm{R}$ rats.

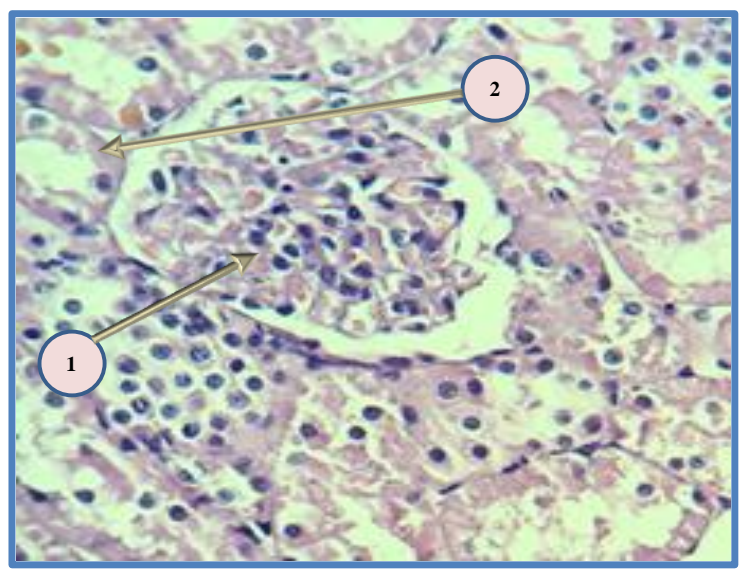

\section{Figure 5A.}

Section through kidney (sham group) showing normal glomerulus (1) and normal renal tubule (2). H and E stain (40X). 


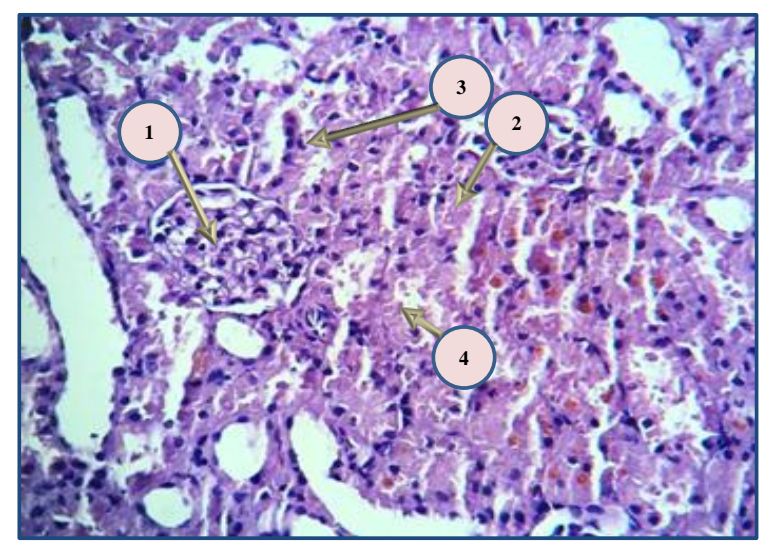

\section{Figure 5B.}

Section through kidney (control untreated I/R group) showing swelling of epithelial cells of glumerulus and narrowing of urinary space (1), increased cytoplasmic eosinophilia and fragmentation (2) and nuclear kayorhexis (4) of renal tubular cells together with nutrophilic inflammatory infiltrate (3). $\mathrm{H}$ and E stain (40X).

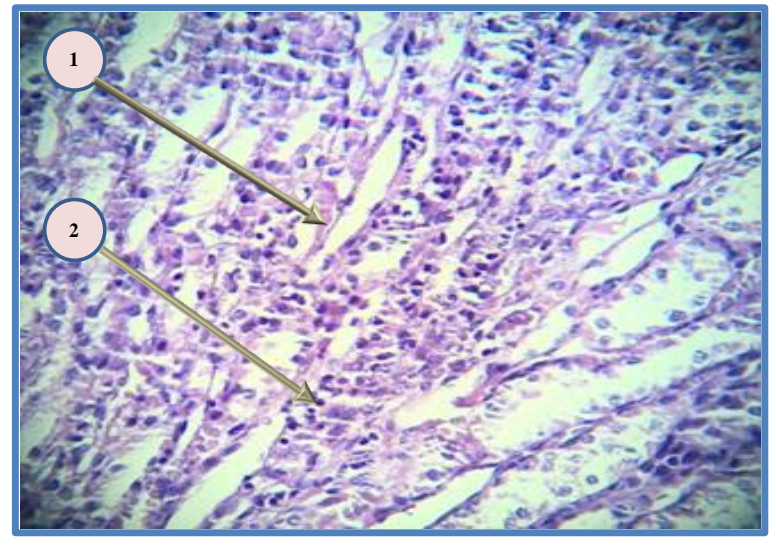

Figure 5C.

Section through kidney (DMSO treated I/R group) nuclear fragmentation (karyorhexis) (2) andrenal tubule cell cytoplasmic eosinophilia and degeneration (1). H and E stain (40X). 


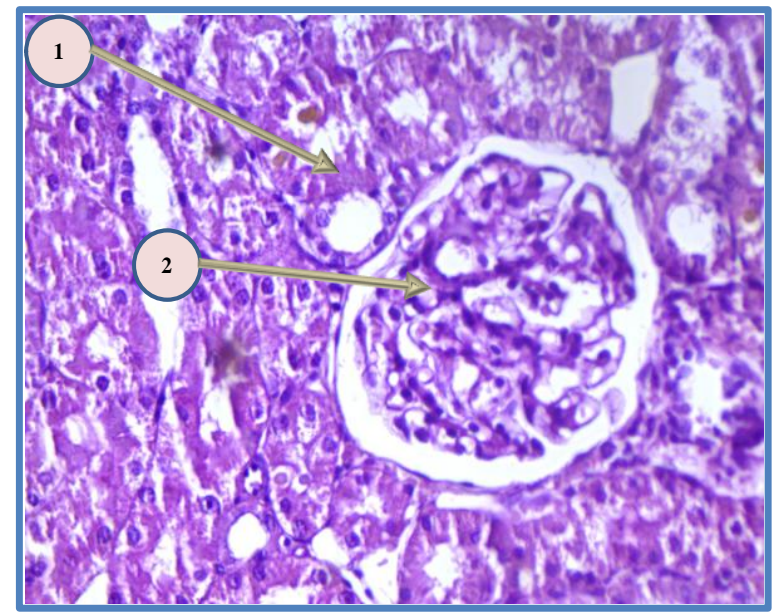

\section{Figure 6A.}

Section through kidney (GIT 27 treated I/R group) showing nuclear fragmentation (karyorhexis) and increased renal tubule cell cytoplasmic eosinophilia (1) and another near normal renal tubule (2). $\mathrm{H}$ and E stain (40X).

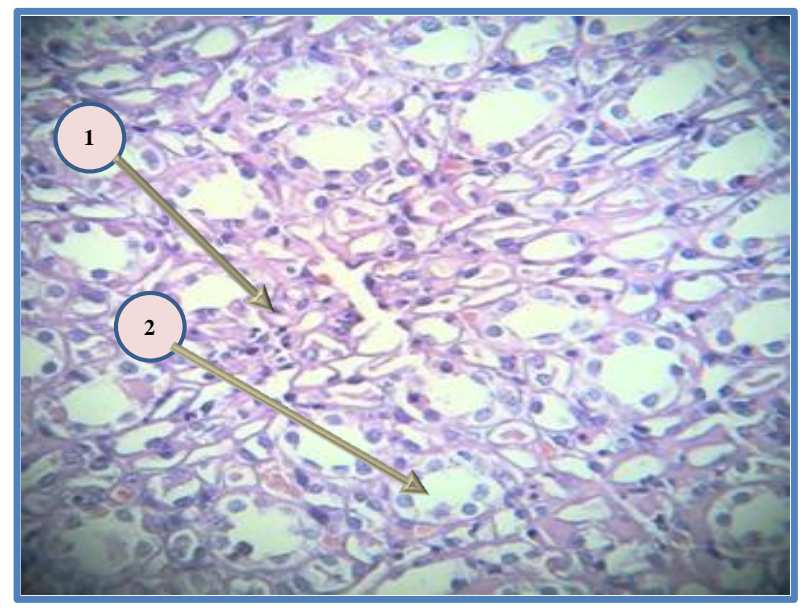

Figure 6B.

Section through kidney (GIT 27 treated I/R group) showing nuclear fragmentation (karyorhexis) and increased renal tubule cell cytoplasmic eosinophilia (1) and another near normal renal tubule (2). $\mathrm{H}$ and E stain (40X).

\section{Discussion}

During renal $\mathrm{I} / \mathrm{R}$, the inflammatory responses mediate renal injury, including dysfunction and renal pathophysiological changes. It is important to create novel medications to protect kidney from IRI [17]. Previous studies reported that expression of inflammatory mediators (TNF- $\alpha$, IL-18) was higher following renal injury $[18,19]$. The present study investigated the GIT-27 to improve the renal function following ischemia reperfusion injury. According to our knowledge there was no data published 
discussed the relationship renal ischemia reperfusion injury and effective role of GIT-27 on improved renal function following $\mathrm{I} / \mathrm{R}$ model in rat. A number of published paper have investigated and confirmed that renal dysfunction during ischemia reperfusions related with inflammatory mediator's expression, including TNF- $\alpha$ and IL-1 $\beta$ [19]. Interestingly, we observed in this study that pretreatment with GIT-27 results in significantly reduction in cytokines with improvement in renal function. The results revealed plasma NGAL was significantly higher in control than that in sham group $(\mathrm{P}<0.05)$ and reduced plasma level of NGAL in GIT-27 pretreated group. Many clinical studies have shown that increases in urinary and plasma NGAL are powerful and independent predictors of AKI when compared with serum creatinine [20, 21, 22]. Clearly, NGAL represents to a novel predictive biomarker for AKI and its results. To best of our knowledge there is no research has measured this parameter regarding the use of GIT-27 against renal ischemia reperfusion injury. However, [23] up regulation of NGAL in renal tubule cells may be induced by local release of cytokines from monocytes in the microcirculation after ischemic injury [24] Nils and others [25] demonstrated that in vascular smooth muscle cells NGAL expression is induced in response to vascular injury and depends on nuclear factor kappa B (NF-kB) expression [26], and upon activation of Toll-like receptors (TLRs) on immune cells establishing an acute phase response [27]. In I/R group, generally all intersections of the most of rat sections of this group indicated tubular cell swelling, brush border loss, nuclear condensation, with more than $66 \%$ of the tubular profile demonstrating nuclear loss. Also intersections of rat sections of GIT-27 pretreated group showed significant improvement in kidney Parenchyma I/R causes significant elevation $(\mathrm{P}<0.05)$ in serum TNF- $\alpha$, tissue IL-18 and which are proinflammatory cytokines that cause adhesion, activation, and transmigration of polymorph nuclear leukocytes (PMNs) into renal tissues and their oxidative burst, which results in excessive ROS production and kidney damage (neutrophil-mediated tissue injury ). Data available in present study showed significant reduction in serum TNF- $\alpha$, tissue IL-18. Cong and others [28] demonstrated that $\mathrm{I} / \mathrm{R}$ in rat model revealed morphologic variations from the norm including cytoplasmic vacuolization, cell necrosis of the proximal convoluted tubule, and tubular lumen obstruction and impairments were discovered generally in I/R group. Recently, researchers showed less albuminuria, mesangial expansion, infiltration of macrophages and proinflammatory and extracellular matrixassociated gene expression in glomeruli as histological changes by GIT-27 [29].

In conclusion, this work found that Toll-like receptor, have critical role in renal $\mathrm{I} / \mathrm{R}$ and lead to attenuated renal function. Further, it was found that Toll-like receptor antagonist (GIT-27) has valuable Reno-protective role related to the changes of pro inflammatory mediators (TNF- $\alpha$, and IL-18), further reduced renal function, and all of these suggest that Toll-like receptor could be a novel target for 
therapy in patients with acute kidney injury induced ischemia reperfusion lead to improved renal function. These experimental results let us believe that Toll-like receptor deactivation led to decreased level NGAL with sequential signal caused renal cell injury.

\section{Competing interests}

The authors declare that there is no conflict of interest.

\section{References}

1. Munshi R, Hsu C, Himmelfarb J. Advances in understanding ischemic acute kidney injury. Bmc Med 2011;9:11.

2. Nash K, Hafeez A, Hou S. Hospitalacquired renal insufficiency. Am $j$ kidney dis 2002;39:930-936.

3. Aragno M, Cutrin JC, Mastrocola R, et al. Oxidative stress and kidney dysfunction due to ischemia/reperfusion in rat: Attenuation by dehydroepiandrosterone. Kidney Int 2003;64:153-158.

4. Wang Jc, Zhao Y, Chen $\mathrm{Sj}$, et al. AOPPs induce MCP-1 expression by increasing ROS-mediated activation of the NF-kappa B pathway in rat mesangial cells: Inhibition by sesquiterpene lactones. Cell Physiol Biochem 2013;32:1867-1877.

5. Bonventre JV, Weinberg JM. Recent advances in the pathophysiology of ischemic acute renal failure. $J A m$ SocNephrol 2003;14(1):2199-2210.

6. Supavekin S, Zhang W, Kucherlapati R, KaskelFj, Moore Lc, Devarajan P. Differential gene expression following early renal ischemia/reperfusion. Kidney Int 2003;63(1):1714-1724.

7. Ozturk H, Ozturk H, Terzi Eh, Ozgen U, Duran A, Uygun I. Protective effects of rosmarinic acid against renal ischemia/reperfusion injury in rats. $J$ Pak Med 2014;64:260-265.

8. Ye S, Zhu Y, Ming Y, She X, Liu H, Ye Q. Glycyrrhizin protects mice against renal ischemia-reperfusion injury through inhibition of apoptosis and inflammation by down regulating p38 mitogen-activated protein kinase signaling. ExpTher Med 2014;7(1):1247-1252.

9. Li Y w, Zhang Y, Zhang L, et al. Protective effect of tea polyphenols on renal ischemia/reperfusion injury via suppressing the activation of TLR4/NF-kappaB p65 signal pathway. Gene 2014;542:46-51.

10. $\mathrm{Hu} \mathrm{H}$, Jiang W, Xi X, Zou C, Ye Z. MicroRNA-21 attenuates renal ischemia reperfusion injury via targeting caspase signaling in mice. Am $J$ Nephrol 2014;40:215-223.

11. Bu Dx, Hemdahl Al, Gabrielsen A, et al. Induction of neutrophil gelatinaseassociated lipocalin in vascular injury via activation of nuclear factor-kappaB. Am J Pathol 2006;169(6):2245-2253.

12. Kawai T, Akira S. TLR signaling. SeminImmunol 2007;19:24-32.

13. Maksimovic-Ivanic D, Mijatovic S, Harhaji $\mathrm{L}$, et al. Anticancer properties of the novel nitric oxide-donating compound (S,R)-3phenyl-4,5-dihydro-5-isoxazole acetic acidnitric oxide in vitro and in vivo. Mol Cancer Ther 2008;7(3):510-20.

14. Hesketh EE, Czopek A, Clay M, Borthwick G, Ferenbach D, Kluth D. Renal ischemia reperfusion injury: a mouse model of injury and regeneration. J. Vis. Exp. 2014;88:e51816.

15. Xie W, Wolin Ms. Role of nitric oxide and its interaction with superoxide in the suppression of cardiac muscle mitochondrial respiration. Involvement in response to hypoxia/reoxygenation. Circulation 1996;94:2580-2586.

16. Mcwhinnie D1, Thompson Jf, Taylor Hm, et al. Morphometric analysis of cellular infiltration assessed by monoclonal antibody labeling in sequential human renal allograft biopsies. ark 1986;42:352-358.

17. Chatterjee Pk. Novel pharmacological approaches to the treatment of renal ischemia-reperfusion injury: A comprehensive review. Arch Pharmacol 2007;376:1-43.

18. Maekawa N, Wada H, Kanda $\mathrm{T}$, et al. Improved myocardial ischemia/reperfusion injury in mice lacking tumor necrosis factoralpha. J Am Coll Cardiol 2002;39(7):122935. 
19. Hadi N, Al-Amran FG, Hussein AA. Effects of thyroid hormone analogue and a leukotrienes pathway-blocker on renal ischemia/reperfusion injury in mice. Nephrol 2011;12:70.

20. Mishra J, Dent C, Tarabishi R, et al. Neutrophil gelatinase associated lipocalin (NGEL)as biomarker for acute kidney injury after cardiac surgery. Lancet 2005;395:1231-8.

21. Haase M, Bellomo R, Devarajan P. Accuracy of neutrophil gelatinaseassociated lipocalin (NGAL) in diagnosis and prognosis in acute kidney injury: a systematic review and meta-analysis. $A m J$ Kidney Dis 2009;54:1012-1024.

22. Paragas N, Qiu A, Hollmen M. NGALsiderocalin in kidney disease. Biochim Biophys Acta 2012;1823:1451-1458.

23. Plewes K, Royakkers AA, Hanson J, et al. Correlation of biomarkers for parasite burden and immune activation with acute kidney injury in severe falciparum malaria. Malar J. 2014;13:91.

24. Mishra J, Ma Q, Prada A, et al. Identification of neutrophil gelatinaseassociated lipocalin as a novel early urinary biomarker for ischemic renal injury. $J \mathrm{Am}$ SocNephrol 2003;14:2534-2543.
25. Trimble CL, Morrow MP, Kraynyak KA. Safety, efficacy, and immunogenicity of VGX-3100, a therapeutic synthetic DNA vaccine targeting human papillomavirus 16 and 18 E6 and E7 proteins for cervical intraepithelial neoplasia 2/3: a randomised, double-blind, placebo-controlled phase $2 \mathrm{~b}$ trial. Lancet 2015;21;386(10008):2078-88.

26. Draper Dw, BetheaHn, He Yw. Toll-like receptor 2-dependent and -independent activation of macrophages by group B streptococci. ImmunolLett 2006;102(2):202214.

27. Lin M,Tang S. Toll-like receptors: sensing and reacting to diabetic injury in the kidney. Oxford Journals Medicine \& Health Nephrology Dialysis Transplantation 2013;29(4):746-754.

28. Chen CC, Liu ZM, Wang HH, He W, Wang $\mathrm{Y}, \mathrm{Wu}$ WD. Effects of ulinastatin on renal ischemia-reperfusion injury in rats. Pharmacol Sin 2004;25(10):1334-1340.

29. Magnusson NE, Hornum M, Jørgensen KA. Plasma neutrophil gelatinase associated lipocalin (NGAL) is associated with kidney function in uremic patients before and after kidney transplantation. BMC Nephrology 2012;13(8).

Editor-In-Chief

Amit Kumar Tyagi MSc, PhD

Department of Experimental Therapeutics

The University of Texas MD Anderson Cancer Center,

1515 Holcombe Blvd

Houston TX 77054, USA

Tele: $713-792-6543$

281-716-0484

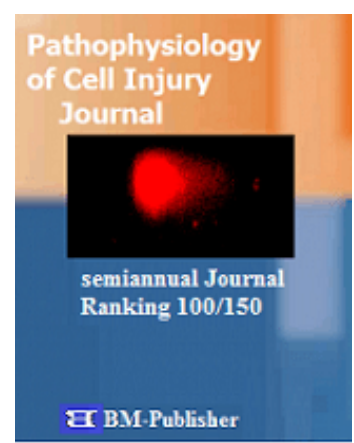

Pathophysiology of Cell Injury Journal "PCIJ" is a semiannual peer-reviewed journal of high priority research, with optional access. PCIJ is a not-for-profit charitable Journal listed and follows the ICMJE's Recommendations for the Conduct, Reporting, Editing and Publication of Scholarly Work in Medical Journals. PCIJ is considered an original articles covers the latest developments in the multidisciplinary areas of the cell injury with its high quality content. PCIJ reflects the field and is an agent for advancing it through critical reviews and news features, consensus documents and guidelines. 
All articles submitted to Pathophysiology of Cell Injury Journal must be original; the work, or large parts of it, must not have been published previously or be currently under consideration or review elsewhere. If there is any significant overlap with another paper, this must be cited in the article and mentioned on submission. All articles are checked for plagiarism on submission; if plagiarism (including self-plagiarism) is identified, the article will be rejected. Submitted articles with content that infringes copyright may be rejected if the problematic sections cannot be removed. Authors who wish to reproduce a figure or table from a previous copyrighted publication are responsible for obtaining the permission of copyright holders and for clearly referencing the original source. 